\title{
NOUVELLE
}

\section{Thérapie par ARN anti-sens dans la maladie de Huntington}

\section{Un immense espoir et beaucoup d'inconnues}

Alexandra Durr
Sorbonne Université, Institut du cerveau et de la moelle épinière (ICM), AP-HP, Inserm, CNRS, Hôpital Pitié-Salpêtrière, 47-83 boulevard de l'Hôpital, 75013 Paris, France.

alexandra.durr@icm-institute.org
> Les résultats de la première tentative de thérapie génique dans une maladie neurologique génétique à révélation tardive ont été publiés dans le numéro $\mathrm{du}$ New England Journal of Medicine du 6 mai 2019. Sous la direction du Pr Sarah Tabrizi (University College London), un essai thérapeutique de phase I-Ila impliquant neuf centres situés en Angleterre, en Allemagne et au Canada, a testé l'innocuité et l'efficacité d'un traitement par oligonucléotide anti-sens visant à diminuer l'expression de la protéine huntingtine (HTT), dont une forme mutée est responsable de la maladie de Huntington [1]. C'est un pari réussi, car cet oligonucléotide complémentaire d'une séquence de 20 nucléotides de I'ARN messager codant la protéine, administré par voie intrathécale à un stade précoce de la maladie, a réduit d'une manière dose-dépendante la concentration de la protéine mutée dans le liquide céphalorachidien des patients.

La maladie de Huntington est une maladie neurologique de transmission autosomique dominante. Elle comporte des troubles psychiatriques, cognitifs et comportementaux, ainsi que des mouvements anormaux (chorée, dystonie), qui s'aggravent progressivement et peuvent constituer un fardeau transmis de génération en génération. Elle est due dans $90 \%$ des cas, à une expansion anormale d'un trinucléotide CAG répété dans le premier exon du gène $H T T$ codant la huntingtine [2]. Le nombre de répétitions CAG des allèles normaux est inférieur à 30 , tandis que les allèles mutés des patients et des personnes à risque de développer la maladie comportent au moins
36 répétitions $C A G$, et entre 40 et 50 dans la majorité des cas. II existe une corrélation inverse entre le nombre de CAG, traduits en une séquence polyglutamine (poly $Q$ ) dans la partie $\mathrm{NH}_{2}$-terminale de la protéine, et l'âge de début, la sévérité clinique et l'intensité des lésions neuropathologiques, avec cependant une importante variation inter-individuelle [3].

La maladie de Huntington fait partie des maladies avec formation d'inclusions intranucléaires qui, dans ce cas, contiennent la protéine HTT ubiquitinylée et hyperphosphorylée. L'accumulation intranucléaire d'une protéine avec expansion du motif poly 0 caractérise également certaines ataxies spino-cérébelleuses et l'atrophie dentato-rubro-pallido-luysienne. L'ARNm du gène HTT est traduit en une protéine soluble, d'une masse moléculaire d'environ $350 \mathrm{kDa}$. Le motif poly 0 de la protéine HTT n'est présent que chez les vertébrés [4], et son rôle dans l'activité de la protéine est encore mal compris. Néanmoins, des souris mutantes comportant une délétion de la région codant le motif polye (constitué par 7 résidus glutamine) vivent plus longtemps, et ont une augmentation de l'autophagie neuronale, suggérant que cette région de la protéine peut moduler la longévité $[5,6]$. L'expansion anormale du motif poly 0 change la conformation de cette région en un feuillet $\beta$, et pourrait ainsi modifier certaines interactions protéiques [7].

Cet essai thérapeutique cible pour la première fois directement la protéine $\mathrm{HTT}$, et suscite donc un espoir important. II s'agit d'une étude randomisée, en double aveugle, réalisée chez 46 patients âgés de 25 à 65 ans, à un stade précoce de la maladie, dont 12 ont été traités par un placebo (liquide céphalo-rachidien artificiel), et 34 par l'oligonucléotide anti-sens thérapeutique $\left(H T_{\mathrm{Rx}}\right)$ administré par voie intrathécale à raison de 4 injections à 4 semaines d'intervalle. Ces 34 patients ont été répartis en cinq sous-groupes selon la dose d'oligonucléotide administrée: 10, 30, 60, 90, ou $120 \mathrm{mg}$ par injection. Un échantillon de liquide céphalo-rachidien a été prélevé avant chaque injection, ainsi que 4 semaines ou 8 semaines après la dernière injection, pour une mesure de la concentration de la protéine HTT mutée (Figure 1). Trois résultats ont ainsi été obtenus.

Premier résultat: chez les patients traités par l'oligonucléotide, la concentration de la protéine mutée dans le liquide céphalo-rachidien diminue de $20 \%$ à $42 \%$ en moyenne selon la dose administrée, tandis que chez les patients ayant reçu le placebo, la protéine mutée s'accumule progressivement (+10\%).

Second résultat: le critère principal d'innocuité est validé car les évènements indésirables survenus chez les patients au cours de cette étude étaient tous mineurs : douleur pendant, ou céphalées après la ponction lombaire, spontanément résolutives en quelques jours.

Troisième résultat: la concentration de la protéine HTT mutée dans le liquide céphalo- rachidien est corrélée au score clinique cUHDRS ${ }^{1}$.

\footnotetext{
1 Le score clinique combiné cUHDRS (composite unified Huntington disease rating scale) évalue la motricité, la cognition et la capacité fonctionnelle globale chez les patients. Il est utilisé pour suivre la progression de la maladie.
} 


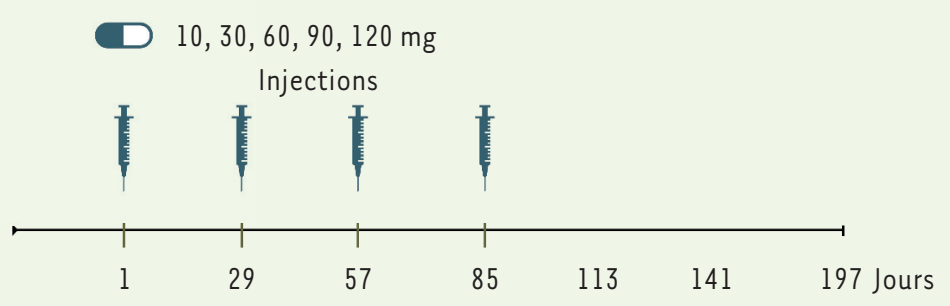

$\lambda$

$\mathrm{n}=46$, $34 \mathrm{HTT}_{\mathrm{Rx}}$

(SM $22 \pm 10)$

12 placebo

$($ SM $24 \pm 7)$
- Espoir : diminution de 20 à $42 \%$ de la concentration de la protéine HTT mutante, en fonction de la dose d'oligonucléotide antisens administrée, dans le liquide céphalorachidien (augmentation de $10 \%$ pour le placebo).

- Traitement et procédure bien tolérés. Inconnues : chez les patients traités à la dose de 90 ou $120 \mathrm{mg}$, le volume ventriculaire estimé par l'IRM cérébrale et la concentration de la chaîne légère des neurofilaments (NfI) dans le LCR ont augmenté (jour 113).

Figure 1. Schéma de l'essai thérapeutique de phase I-Ila, randomisé et en double aveugle, réalisé à un stade précoce de la maladie de Huntington. Le traitement par un oligonucléotide antisens $\left(\mathrm{HTT}_{\mathrm{Rx}}\right)$ a été administré à 34 patients, en 4 injections par voie intrathécale, à 4 semaines d'intervalle, à des doses variant de 10 à $120 \mathrm{mg}$ par injection selon le sous-groupe de patients ( 5 sous-groupes comportant de 3 à 10 patients chacun), tandis que 12 patients ont reçu un placebo (liquide céphalo-rachidien artificiel). La seringue symbolise chaque injection. Un prélèvement supplémentaire de liquide céphalo-rachidien a été effectué 4 semaines ou 8 semaines après la dernière injection, au jour 113 ou 14l. L'oligonucléotide anti-sens $\mathrm{HTT}_{\mathrm{Rx}}$ a été conçu pour inhiber l'ARN messager du gène HTT, et ainsi réduire la concentration intracellulaire de la protéine huntingtine (HTT). SM : score moteur (de 0 à 124), dont la valeur chez les patients augmente avec la sévérité de la maladie; : dose de $\mathrm{HTT}_{\mathrm{Rx}}$ ou placebo.

Il reste néanmoins plusieurs incertitudes, qu'il faudra s'efforcer de lever dans l'essai de phase III qui a débuté cet été dans plus de 80 centres, sur 660 patients au total ${ }^{2}$. D'abord, une augmentation du volume des ventricules cérébraux, dépendante de la dose d'oligonucléotide administrée, a été observée par imagerie par résonance magnétique (IRM). Elle suggère que l'atrophie cérébrale sous traitement à forte dose est plus importante qu'attendu. De plus, cette étude a mis en évidence une élévation transitoire de la concentration de la chaîne légère des neurofilaments ( $\mathrm{NFI}$ ) dans le liquide céphalo-rachidien, 4 ou 8 semaines après la dernière injection, chez les patients ayant reçu de fortes doses de l'oligonucléotide (90 ou $120 \mathrm{mg}$ ). $\mathrm{Nfl}$ est un marqueur non spécifique,

\footnotetext{
${ }^{2}$ https://clinicaltrials.gov/ct2/show/NCT03761849
}

dont la concentration mesurée dans le sérum ou dans le liquide céphalo-rachidien est corrélée avec la sévérité de la maladie dans plusieurs maladies neurodégénératives, notamment la sclérose latérale amyotrophique ou la maladie d'Alzheimer. Une augmentation de la concentration de cette protéine pourrait indiquer une destruction axonale ou une perte neuronale. Les conséquences de la diminution de la concentration cellulaire de la protéine HTT normale induite par le traitement ne sont pas non plus connues. La maladie de Huntington est due à une mutation présente à l'état hétérozygote entraînant un effet dominant, avec une accumulation de la protéine mutée, mais aussi une perte ou une modification de l'activité normale de la protéine [8]. La protéine HTT intervient dans de nombreux processus cellulaires (transcription génique, transport intracellulaire, cilio- genèse, autophagie, endocytose, etc.), du fait de la multitude de ses partenaires de liaison (environ 500) et de ses localisations subcellulaires variées. Son rôle dans la division cellulaire et le cancer reste à élucider $[9,10]$, ainsi que dans le développement cérébral précoce [11]. L'oligonucléotide anti-sens $\mathrm{HTT}_{\mathrm{Rx}}$ administré aux patients de l'étude n'est pas spécifique de l'allèle muté, et on ne sait pas encore si la diminution de la concentration cellulaire de la protéine HTT normale a des conséquences néfastes à long terme. Enfin, il reste surtout à évaluer l'effet clinique de ce traitement, ce qui n'était pas l'objectif de cet essai de phase I-Ila. Observera-t-on une amélioration apparente des symptômes de la maladie, et dans l'affirmative, portera-t-elle sur les troubles moteurs ou psychiatriques? Part ailleurs, le stade idéal pour entreprendre le traitement (a priori plutôt aux stades précoces de la maladie, voire au stade pré-symptomatique) et sa durée optimale restent à déterminer. Quoi qu'il en soit, cette promesse d'un traitement direct de la cause de la maladie de Huntington ouvre assurément une nouvelle ère thérapeutique pour les maladies neurogénétiques de révélation tardive. $\diamond$

Anti-sense oligonucleotides RNA therapy in Huntington disease: a great promise and many unknowns

\section{LIENS D'INTÉRÊT}

Alexandra Durr est investigatrice de l'essai de phase III mené par Roche et est consultante pour Wavelife et Triplets therapeutics.

\section{RéFÉRENCES}

1. Tabrizi S, Leavitt B, Landwehrmeyer B, et al. Targeting huntingtin expression in patients with Huntington's disease. N Engl J Med 2019 ; 380 : 2307-16.

2. Mariani LL, Tesson C, Charles P, et al. Expanding the spectrum of genes involved in Huntington disease using a combined clinical and genetic approach. JAMA Neurol 2016 ; 73 : 1105-14.

3. Cazeneuve C, Durr A. Huntington's disease: genetic and molecular studies. In : Bates G, Tabrizi S, Jones L, eds. Huntington's disease. Oxford: Oxford University Press, 2014 : 109-31.

4. Zuccato C, Valenza M, Cattaneo E. Molecular mechanisms and potential therapeutical targets in Huntington's disease. Physiol Rev $2010 ; 90: 905-81$. 


\section{RÉFÉRENCES}

5. Clabough EB, Zeitlin SO. Deletion of the triplet repeat encoding polyglutamine within the mouse Huntington's disease gene results in subtle behavioral/motor phenotypes in vivo and elevated levels of ATP with cellular senescence in vitro. Hum Mol Genet 2006 ; $15: 607-23$.

6. Zheng $S$, Clabough $\varepsilon B$, Sarkar $S$, et al. Deletion of the huntingtin polyglutamine stretch enhances neuronal autophagy and longevity in mice. PLOS Genet $2010 ; 6$ : el000838

7. Darnell G, Orgel JP, Pahl R, Meredith SC. Flanking polyproline sequences inhibit $\beta$-sheet structure in polyglutamine segments by inducing PPII-like helix structure. J Mol Biol 2007 ; 374 : 688-704.

8. Saudou F, Humbert $S$. The biology of huntingtin. Neuron $2016 ; 89$ : 910-26.

9. Thion MS, Tézenas du Montcel S, Golmard JL et al. CAG repeat size in huntingtin alleles is associated with cancer prognosis. EurJ Hum Genet 2016 ; 24 : 1310-5.
10. Coarelli G, Diallo A, Thion MS, et al. Low cancer prevalence in polyglutamine expansion diseases. Neurology 2017 ; 88 : 1114-9.

11. Barnat M, Le Friec J, Benstaali C, Humbert S.

Huntingtin-mediated multipolar-bipolar transition of newborn cortical neurons is critical for their postnatal neuronal morphology. Neuron 2017 ; 93 : 99-114. 